\title{
3D nongadolinium-enhanced mra using flow-sensitive dephasing (fsd) prepared balanced ssfp: identification of the optimal first-order gradient moment

\author{
Zhaoyang Fan*1, Xiaoming Bi², Xiangzhi Zhou ${ }^{1}$, Sven Zuehlsdorff2, \\ James Carr ${ }^{1}$ and Debiao $\mathrm{Li}^{1}$
}

Address: ${ }^{1}$ Northwestern University, Chicago, IL, USA and ${ }^{2}$ Siemens Medical Solutions, Chicago, IL, USA

* Corresponding author

from 13th Annual SCMR Scientific Sessions

Phoenix, AZ, USA. 2I-24 January 2010

Published: 21 January 2010

Journal of Cardiovascular Magnetic Resonance 20 I0, I2(Suppl I):O65 doi:10.1 I86/1532-429X-12-SI-O65

This abstract is available from: http://jcmr-online.com/content/I2/SI/O65

(C) 2010 Fan et al; licensee BioMed Central Ltd.

\section{Introduction}

A noncontrast MRA technique using ECG-triggered 3D bSSFP with FSD preparation has recently been developed (Fig. 1) [1]. The angiographic quality relies on the blood signal suppression by FSD preparation, which is in turn determined by FSD's first-order gradient moment, $\mathrm{m}_{1}$. A suboptimal $\mathrm{m}_{1}$ may result in venous contamination or incomplete arterial delineation. This work aimed to develop an $\mathrm{m}_{1}$-scout approach to rapidly identify the optimal $\mathrm{m}_{1}$-value.

\section{Materials and methods \\ Theory}

For a FSD-based 3D isotropic resolution MRA with major flows along the readout direction, we hypothesized that the same imaging sequence can be switched to a 2D mode to rapidly identify the optimal $m_{1}$ as applied in the readout direction during the $3 \mathrm{D}$ dark-artery measurement. This requires the $2 \mathrm{D}$ imaging plane be perpendicular to the major vessel of interest, the FSD gradient pulses applied in the slice-select direction, and the in-plane resolution identical to that of 3D MRA.

\section{Imaging}

(1) Flow phantom study (Gd-doped water $0.25 \mathrm{mM}$ ). Six flow rates $(15,20,30, \ldots 60 \mathrm{~cm} / \mathrm{s})$ were tested. A 2D FSDbSSFP scan, as $\mathrm{m}_{1}$-scout, acquired 11 images with incremental $\mathrm{m}_{1}$ values (0.9-mm in-plane and 5-mm slice-thickness) (Fig. 2). 3D FSD-bSSFP imaging was repeated with 6 selected $\mathrm{m}_{1}$-values (0.9-mm isotropic). (2) Volunteer study ( $3 \mathrm{M}, 2 \mathrm{~F})$. Left and right thighs were scanned separately. $2 \mathrm{D} \mathrm{m} \mathrm{m}_{1}$-scout imaging employed $\mathrm{m}_{1}=0,5, \ldots 50$ $\mathrm{mT} \cdot \mathrm{ms}^{2} / \mathrm{m}$, respectively. 3D FSD-bSSFP imaging was repeated with 7 selected $m_{1}$-values.

\section{Results}

The $2 \mathrm{D}$ scout took $<1 \mathrm{~min}$ as opposed to $4-5 \mathrm{~min}$ required for 3D MRA. In the phantom study, the lumen signal intensity from the 2D and 3D images were significantly correlated at all the velocities tested (Pearson correlation $=0.988 \pm 0.011, \mathrm{p}<0.001)$ (Fig. 3). Similar results were observed in the volunteer study (Fig. 4a, Table 1).

\section{Conclusion}

The optimal $\mathrm{m}_{1}$ value determined by the $2 \mathrm{D}$ scout approach consistently offers high-quality MRA at the volunteer extremities (Fig. 4b, 5, 6). FSD-induced signal suppression is voxel size-dependent as its underlying mechanism is the intravoxel velocity variation $[1,2]$. Despite the large pixel size in the 2D scout, this variation is equivalent between the $2 \mathrm{D}$ and $3 \mathrm{D}$ scans if the velocity is constant along the vessel course. This efficient approach is likely to be beneficial for FSD-based MRA and vessel wall imaging [3]. 

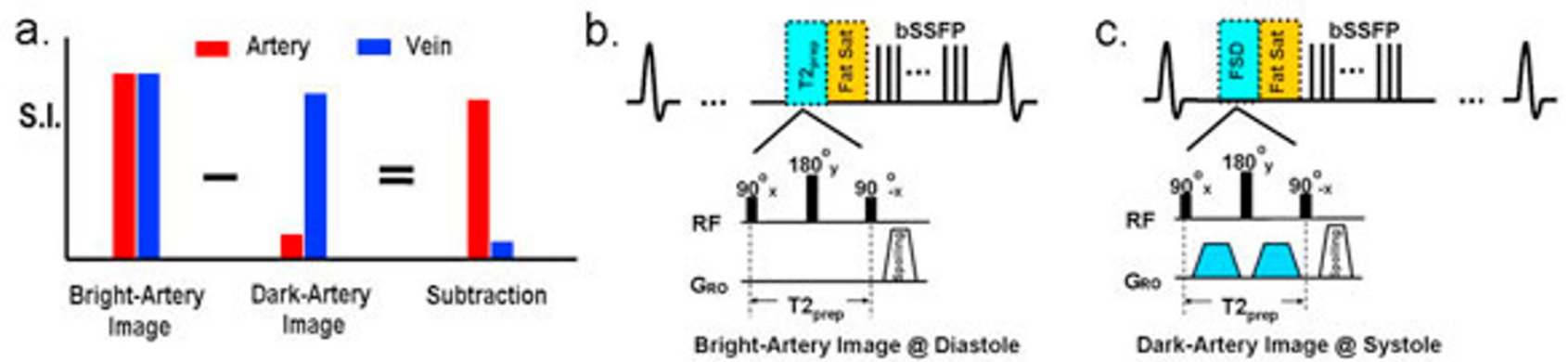

Figure I

Schematic of FSD-prepared MRA method (a) and sequence diagrmas (b and c).

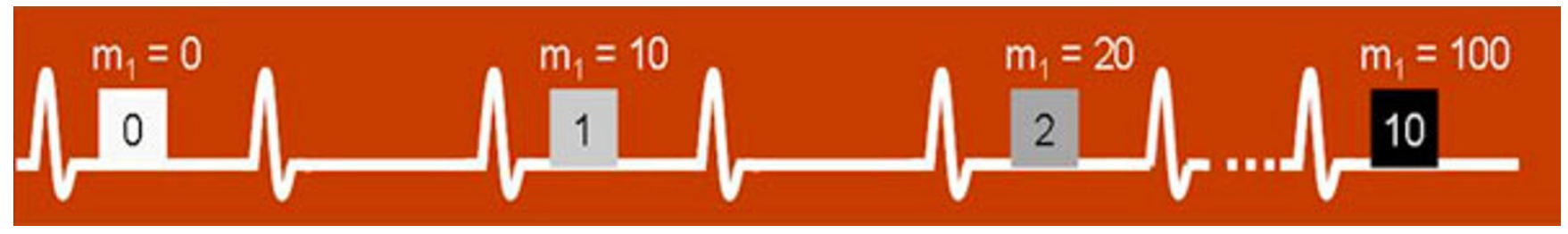

\section{Figure 2}

Schematic of the 2D $\mathrm{m}_{1}$-scout approach. A total of II images were collected within I min. The first uses $\mathrm{m}_{\mathrm{I}}=0$, while the latter uses incremental $m_{1}$ values (user specified)

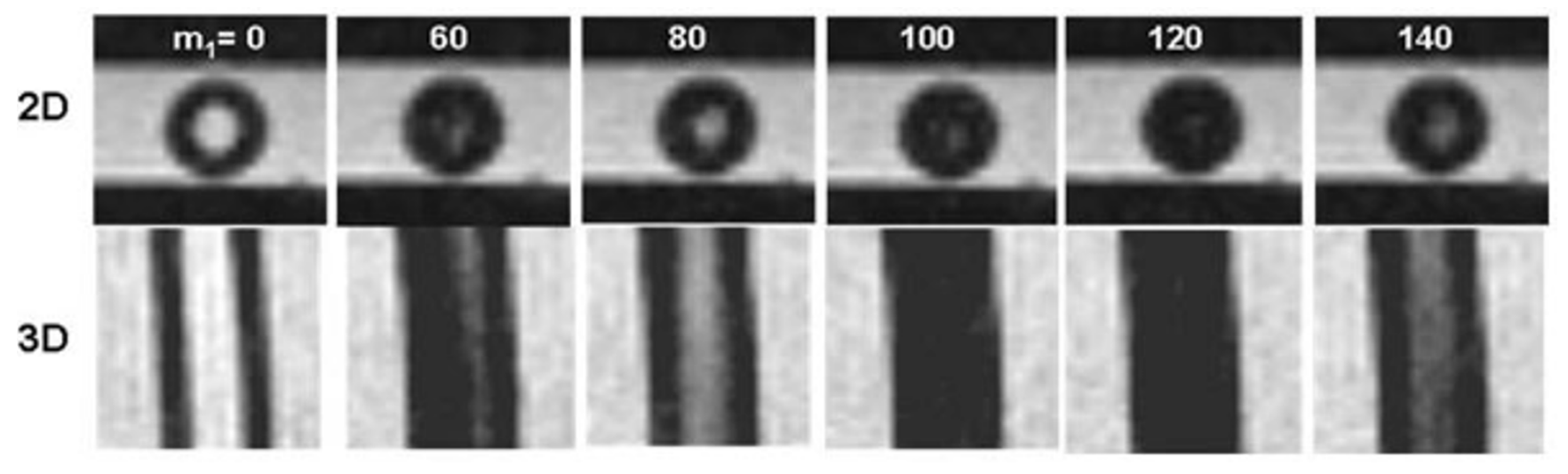

Figure 3

2D scout vs. 3D reformatted images (flow phantom).

Table I: Signal intensity Pearson correlation in $\mathbf{5}$ healthy volunteers

\begin{tabular}{lllllllllll}
\hline Volunteer (Left/Right) & $\mathbf{I}$ & $\mathbf{I}$ & $\mathbf{2}$ & $\mathbf{2}$ & $\mathbf{3}$ & $\mathbf{3}$ & $\begin{array}{l}\mathbf{4} \\
\mathbf{( L )}\end{array}$ & $\begin{array}{l}\mathbf{4} \\
\mathbf{( R )}\end{array}$ & $\begin{array}{l}\mathbf{5} \\
\mathbf{( L )}\end{array}$ & $\begin{array}{l}\mathbf{5} \\
\mathbf{( R )}\end{array}$ \\
\hline $\begin{array}{l}\text { Pearson Correlation } \\
p \text {-value }\end{array}$ & 0.941 & 0.955 & 0.974 & 0.984 & 0.990 & 0.992 & 0.947 & 0.960 & 0.924 & 0.950 \\
\hline
\end{tabular}


a.

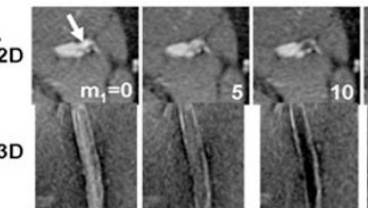

b.

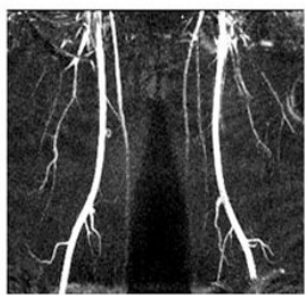

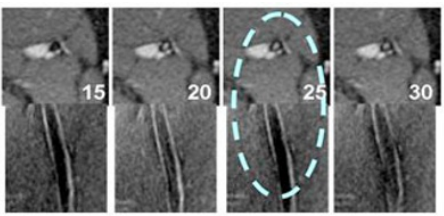

Fig 4. 2D scout vs. 3D reformatted images of superficial femoral arteries from a volunteer (a) and MRA using the optimal $m_{1}$-value $\left(15 \mathrm{mT} \cdot \mathrm{ms}^{2} / \mathrm{m}\right)$. There is a discrepancy at $\mathrm{m}_{1}=25 \mathrm{mT} \cdot \mathrm{ms}^{2} / \mathrm{m}$ (dashed ellipse), likely due to the altered flow pattern or ECG timing.

Figure 4

2D scout vs. 3D reformatted images of superficial femoral arteries from a volunteer (a) and MRA using the optimal $m_{1}$-value $\left(15 \mathrm{mT} \mathrm{ms}^{2} / \mathrm{m}\right)$. There is a discrepancy at $\mathrm{m}^{\mathrm{l}}=25 \mathrm{mT} \mathrm{ms}^{2} / \mathrm{m}$ (dashed ellipse), likely due to the altered flow pattern or ECG timing

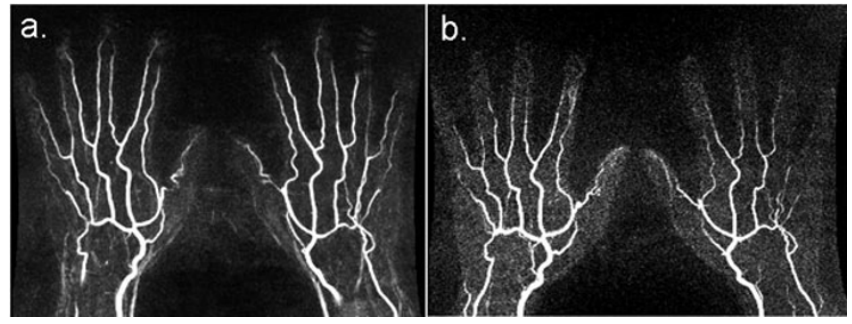

Figure 6

Noncontrast hand MRA (a) has better arterial depiction than contrast MRA (b).

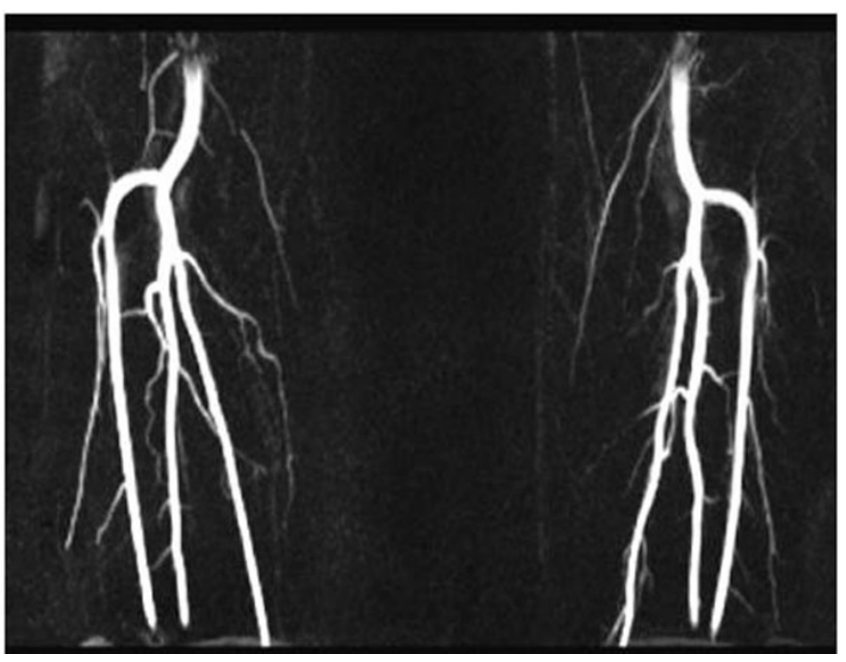

Figure 5

Superior arterial depiction in calf MRA. 